\title{
SLEEP-DISORDERED BREATHING
}

\section{Effect of CPAP on insulin resistance and $\mathrm{HbAlc}$ in men with obstructive sleep apnoea and type 2 diabetes}

\section{Sophie D West, Debby J Nicoll, Tara M Wallace, David R Matthews, John R Stradling}

Thorax 2007;62:969-974. doi: 10.1136/thx.2006.074351

See end of article for authors' affiliations

\section{Correspondence to:} Dr Sophie D West, Sleep Unit, Oxford Centre for Respiratory Medicine Churchill Hospital, Old Road, Oxford OX3 7ป, UK; sophie@west66.freeserve. co.uk

Received 2 November 2006 Accepted 10 April 2007

Published Online First 8 June 2007
Background: The effects of continuous positive airway pressure (CPAP) for obstructive sleep apnoea (OSA) on insulin resistance are not clear. Trials have found conflicting results and no appropriate control groups have been used.

Methods: Forty-two men with known type 2 diabetes and newly diagnosed OSA ( $>10$ dips/h in oxygen saturation of $>4 \%)$ were randomised to receive therapeutic $(n=20)$ or placebo CPAP $(n=22)$ for 3 months. Baseline tests were performed and repeated after 3 months. The study was double blind.

Results: Results are expressed as mean (SD). CPAP improved the Epworth sleepiness score significantly more in the therapeutic group than in the placebo group $(-6.6(4.5)$ vs $-2.6(4.9), p=0.01)$. The maintenance of wakefulness test improved significantly in the therapeutic group but not in the placebo group $(+10.6$ (13.9) vs -4.7 (11.8) $\mathrm{min}, p=0.001$ ). Glycaemic control and insulin resistance did not significantly change in either the therapeutic or placebo groups: $\mathrm{HbAlc}(-0.02(1.5)$ vs $+0.1(0.7), \mathrm{p}=0.7,95 \% \mathrm{Cl}-0.6 \%$ to $+0.9 \%)$, euglycaemic clamp (M/l: +1.7 (14.1) vs -5.7 (14.8), $p=0.2,95 \% \mathrm{Cl}-1.8$ to $\left.+0.3 \mathrm{l} / \mathrm{kg} / \mathrm{min}^{1000}\right)$, HOMA$\% \mathrm{~S}(-1.5(2.3)$ vs $-1.1(1.8), \mathrm{p}=0.2,95 \% \mathrm{Cl}-0.3 \%$ to $+0.08 \%)$ and adiponectin $(-1.1(1.2)$ vs $-1.1(1.3)$, $\mathrm{p}=0.2,95 \% \mathrm{Cl}-0.7$ to $+0.6 \mu \mathrm{g} / \mathrm{ml})$. Body mass index, bioimpedance and anthropometric measurements were unchanged. Hours of CPAP use per night were $3.6(2.8)$ in the treatment group and $3.3(3.0)$ in the placebo group $(p=0.8)$. There was no correlation between CPAP use and the measures of glycaemic control or insulin resistance.

Conclusion: Therapeutic CPAP does not significantly improve measures of glycaemic control or insulin resistance in men with type 2 diabetes and OSA.
O bstructive sleep apnoea (OSA) is characterised by recurrent upper airway obstruction during sleep, recurrent apnoeas and arousals. It is associated with central obesity and affects approximately $4 \%$ of men. ${ }^{1}$ Population studies have found that OSA is associated with insulin resistance, and the more severe the OSA, the greater the insulin resistance, independent of general obesity. ${ }^{2}$ Insulin resistance occurs when the metabolic effect of insulin is reduced, leading to a lack of hepatic and peripheral tissue response to insulin-mediated glucose metabolism. ${ }^{4}$ This most closely correlates with central obesity, and the greater the visceral fat, the greater the insulin resistance. ${ }^{5}$ It is postulated that the insulin resistance in OSA is due, not only to visceral obesity, but also to increased sympathetic drive from the frequent arousals, hypoxia and sleep fragmentation-all of which are thought to impair glucose tolerance. ${ }^{67}$

Insulin resistance is frequently observed in pre-diabetes and type 2 diabetes develops when normoglycaemia is no longer maintained as a result of inadequate pancreatic $\beta$ cell compensation and insulin production. Insulin resistance is affected by many variables including changes in weight, body fat distribution (with visceral fat causing more insulin resistance than subcutaneous fat), exercise, drugs and smoking. Studies of longitudinal change in insulin resistance as an outcome need to include a control group to allow for such confounders. Insulin resistance is measurable by several techniques including the homeostatic model assessment (HOMA) for basal assessment ${ }^{8}$ and the euglycaemic clamp for stimulated insulin assessment. ${ }^{9}$

There has been interest in whether the use of continuous positive airway pressure (CPAP) for the treatment of OSA can improve the insulin resistance found in OSA. If the hypoxia, arousals and increased sympathetic drive found in OSA were adequately treated, would the insulin resistance and hence the glycaemic control improve? Several studies have tried to answer this but, so far, the available data have not led to a conclusive answer as the significance of any changes cannot be assessed without a control group. ${ }^{10-14}$ We therefore performed a randomised controlled trial using therapeutic and placebo CPAP to assess the effect of CPAP on glycaemic control (glycosylated haemoglobin, HbAlc) and insulin resistance (determined by euglycaemic clamp and HOMA) in men with established type 2 diabetes and newly diagnosed OSA.

\section{METHODS \\ Subjects}

Subjects were recruited via the Oxford Sleep Clinic between June 2004 and August 2005. Eligible subjects were men aged 18-75 years with established type 2 diabetes (on diet, oral hypoglycaemic agents or insulin therapy). They had excessive daytime sleepiness (Epworth Sleepiness Score (ESS) $\geqslant 9$ ) and were due to start CPAP for OSA, established from overnight laboratory sleep studies (VisiLab, Stowood Scientific Instruments, Oxford, UK). The entry criterion for OSA was $>10$ oxygen saturation $\left(\mathrm{SaO}_{2}\right)$ dips of $>4 \%$ per hour on an overnight sleep study. Patients were excluded if they required urgent CPAP or if they had unstable diabetes (requiring an escalation in treatment). Additional details are provided in the online data supplement available at http://thorax.bmj.com/ supplemental.

\footnotetext{
Abbreviations: $\mathrm{BMI}$, body mass index; $\mathrm{CPAP}$, continuous positive airway pressure; ESS, Epworth sleepiness score; HbAlc, glycosylated haemoglobin; HOMA, homeostatic model assessment; MWT, Maintenance of Wakefulness test; OHA, oral hypoglycaemic agents; OSA, obstructive sleep apnoea; SAQLI, Sleep Apnea Quality of Life Index
} 


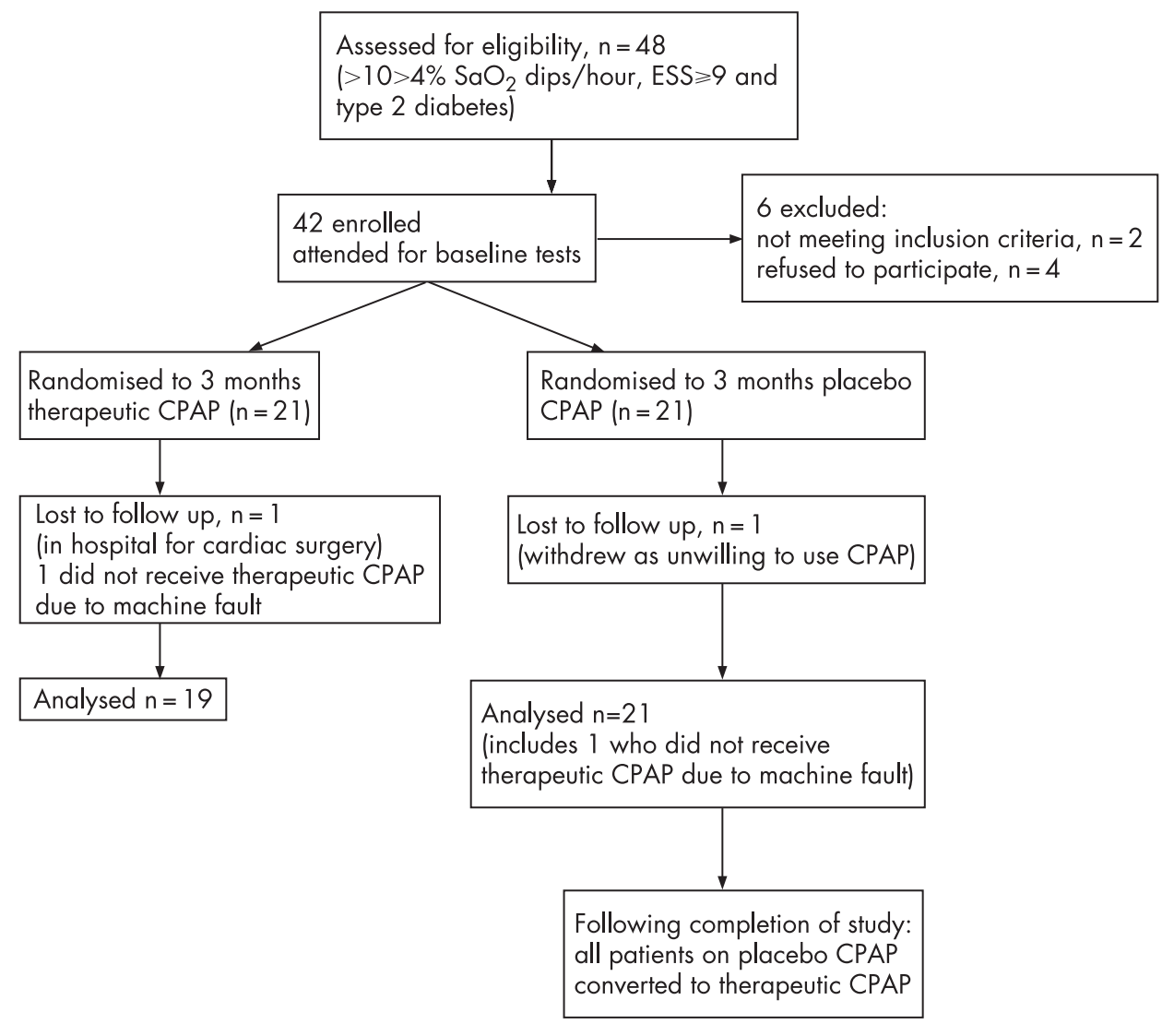

Figure 1 Flow chart of study.

\section{Study design}

Eligible subjects were seen for their baseline study visit 10 days before commencing CPAP. Subjects were asked not to change their diet or exercise habits for the duration of the study, and their primary care physicians were asked not to change their medications unless essential. Following baseline studies, each subject was randomised to receive either therapeutic or placebo CPAP for 3 months in a double blind fashion. Randomisation was by means of a balanced computer programme (MINIM Version 1.5, Evans S). CPAP was first used overnight at home following an afternoon training session, which is standard practice in our unit. Two weeks after CPAP initiation, all patients were seen in the nurse-led CPAP clinic. The nurses involved in the randomisation, CPAP initiation and ongoing CPAP care were separate from the study investigators. After 3 months of CPAP treatment the baseline studies were repeated. At the end of the study all subjects receiving placebo CPAP were changed to therapeutic CPAP. Subjects gave written informed consent and the study was approved by the local ethics committee.

\section{CPAP}

Subjects receiving therapeutic CPAP had autotitrating machines (Autoset Spirit, ResMed, UK) while those receiving placebo CPAP had the same machines set to their lowest pressure with a flow restricting connector inserted at the machine outlet and six extra $4 \mathrm{~mm}$ holes inserted in the collar of the main tubing to allow air to escape and to prevent rebreathing of carbon dioxide. A pressure of $<1$ and $>0 \mathrm{~cm} \mathrm{H}_{2} \mathrm{O}$ was delivered, insufficient to hold open the pharynx. These methods of placebo CPAP provision have been used previously. ${ }^{15}{ }^{16}$ The data from the CPAP machines were downloaded at the second study visit.

\section{Measures of insulin resistance}

Insulin resistance was assessed by both HOMA and euglycaemic hyperinsulinaemic clamp on a single day. Studies were carried out after an overnight fast and omission of the morning oral hypoglycaemic agents or insulin. Baseline blood samples were collected for the determination of glucose and insulin for HOMA. Following the basal sampling, subjects underwent a hyperinsulinaemic euglycaemic clamp. ${ }^{9}$ They were kept awake for the duration of the clamp in order to avoid any confounding effects of sleep on glucose metabolism.

\section{Other blood tests}

HbAlc, lipids (cholesterol, HDL-cholesterol, triglycerides), adiponectin and highly sensitive C-reactive protein were measured.

\section{Measures of body composition}

Height and weight were recorded, body mass index (BMI) was calculated and neck, waist and hip measurements were made. Body composition was measured using bioelectrical impedance analysis (Bodystat 1500, UK).

\section{Measures of sleepiness and activity}

Subjective sleepiness was measured by the ESS and objective sleepiness was measured once at the same time of day using a modification of the Maintenance of Wakefulness test (MWT) (OSLER). ${ }^{17}$ The Short Sleep Apnea Quality of Life Index (Short SAQLI) was completed. ${ }^{18}$ These variables were measured to confirm patients were responding to therapeutic CPAP compared with the placebo group. Physical activity was assessed at baseline and at the end of the study using wrist worn actiwatches (electronic devices containing accelerometers which measure and record intensity, amount and duration of physical movement; Cambridge Neurotechnology Ltd, UK). 


\section{Analysis of data}

The primary end point was the change in HbAlc measured after 3 months of therapeutic or placebo CPAP. Secondary end points were changes in insulin sensitivity measured by HOMA and euglycaemic clamp. Differences between groups (using the change from baseline as the outcome variable) were assessed with the unpaired Student $t$ test. A $\chi^{2}$ test was used to compare the proportions in each group on different diabetes therapy and duration of diabetes. Non-normally distributed data were logarithmically transformed before applying parametric statistical tests and data are reported as geometric means. Activity data were analysed using non-parametric tests. A p value of $<0.05$ was considered to be statistically significant. Analysis was performed with SPSS Version 12.0.

\section{Study size}

The study was powered not to miss a difference of 0.8 in HbAlc (assuming a within subject SD of 0.8$)^{19}$ at a significance level of $5 \%$ and with a power of $90 \%$, which required 20 subjects in each treatment group.

\section{RESULTS}

Figure 1 shows a flow chart of the study. Forty-eight men were considered for entry to the study: four declined and two were unsuitable, so 42 were enrolled. Twenty-one men were randomised to receive therapeutic CPAP and 21 to receive placebo CPAP. One patient randomised to receive therapeutic CPAP had a defective machine which delivered minimal pressure so his data were therefore analysed with the placebo group. The two groups were well matched at baseline with no significant difference in age (mean (range): therapeutic 58 (2974) years, placebo 55 (24-66) years), $>4 \% \quad \mathrm{SaO}_{2}$ dips/hour (therapeutic 33.1 (11.0-87.9), placebo 39.1 (10.8-82.2), BMI (therapeutic $36.6(26.2-49.2)$, placebo $36.8(29.2-47.1)$ ) or HbAlc (therapeutic 8.5 (6.5-12.1), placebo (8.4 (6.0-13.6)). For diabetes, five subjects were treated with diet only, 23 with oral hypoglycaemic agents (OHA), 23 with insulin and OHA, and four with insulin alone; the proportions of subjects receiving these treatment categories were not significantly different between the two groups $\left(p=0.6, \chi^{2}\right.$ test). The mean duration of diabetes was 7.3 years in the therapeutic CPAP group and
6.5 years in the placebo group $\left(p=0.3, \chi^{2}\right.$ test). Completion data were available for 40 men: one patient receiving therapeutic CPAP did not attend his second study visit as he was admitted to hospital for emergency cardiac surgery and one patient withdrew from the study because he was unwilling to continue using CPAP (randomised to placebo). Patients who attended and had poor or negligible CPAP usage were included and analysed on an intention to treat basis. Euglycaemic clamps were performed on 33 of the study participants; technical difficulties meant these were not performed in the first nine participants. There were no adverse events in either of the groups.

\section{Measures of daytime sleepiness and SAQLI score}

Subjective sleepiness measured by the ESS improved in both groups following CPAP treatment (table 1), but the change was significantly greater in the group receiving therapeutic CPAP $(\mathrm{p}<0.01)$. Objective sleepiness, measured by the modified MWT, improved significantly only in the group receiving therapeutic CPAP by a mean of $+10.6 \mathrm{~min}(\mathrm{p}<0.001)$. This change in MWT was similar to our previous randomised controlled trial in this area $(+7.0 \mathrm{~min})$, and the change in ESS was of an effect size (1.9) nearly as large as this previous study (2.2) in which the patients had less comorbidity. ${ }^{15}$ The mean short SAQLI score improved following CPAP treatment in both groups, but the change between the groups was significantly different in favour of therapeutic CPAP $(p=0.04)$.

\section{Clamp characteristics}

The mean (SD) blood glucose concentrations over the last $20 \mathrm{~min}$ of baseline euglycaemic clamp were 5.9 (0.5) $\mathrm{mmol} / \mathrm{l}$ in the therapeutic CPAP group and $5.9(0.5) \mathrm{mmol} / \mathrm{l}$ in the placebo group $(\mathrm{p}=0.8)$; and in the repeat clamp the levels were 6.1 (0.8) $\mathrm{mmol} / \mathrm{l}$ and $5.9(0.5) \mathrm{mmol} / \mathrm{l}$, respectively $(\mathrm{p}=0.5)$.

\section{$\mathrm{HbAlc}$ and insulin sensitivity}

The results are shown in table 1 . HbAlc did not change significantly following CPAP treatment in either of the groups. There was no significant change in insulin sensitivity in either the therapeutic or placebo CPAP groups after 3 months of treatment. The plasma insulin concentrations during the

Table 1 Baseline measurements and changes from baseline in subjects randomised to therapeutic and placebo CPAP

\begin{tabular}{|c|c|c|c|c|c|c|}
\hline & \multicolumn{2}{|l|}{ Baseline } & \multicolumn{2}{|c|}{ Change from baseline $(\Delta)$} & \multirow[b]{2}{*}{$95 \% \mathrm{Cl}$ between groups } & \multirow[b]{2}{*}{ p Value (for $\Delta$ ) } \\
\hline & $\begin{array}{l}\text { Therapeutic CPAP } \\
(n=20)\end{array}$ & $\begin{array}{l}\text { Placebo CPAP } \\
(n=22)\end{array}$ & $\begin{array}{l}\text { Therapeutic CPAP } \\
(n=19)\end{array}$ & $\begin{array}{l}\text { Placebo CPAP } \\
(n=21)\end{array}$ & & \\
\hline Age (years) & $57.8(10.4)$ & $54.5(9.4)$ & & & & \\
\hline$>4 \% \mathrm{SaO}_{2} \mathrm{dips} / \mathrm{h}$ & $33.1(21.6)$ & $39.1(24.8)$ & & & & \\
\hline ESS & 14.7 (3.5) & $13.6(3.5)$ & $-6.6(4.5)$ & $-2.6(4.9)$ & -7.0 to -0.9 & 0.01 \\
\hline MWT (Osler) (min) & $21.9(12.8)$ & $32.0(10.8)$ & $+10.6(13.9)$ & $-4.7(11.8)$ & +6.5 to +23.9 & 0.001 \\
\hline BMI $\left(\mathrm{kg} / \mathrm{m}^{2}\right)$ & $36.6(4.9)$ & $36.8(4.6)$ & $-0.2(1.0)$ & $-0.2(1.1)$ & -0.6 to +0.7 & 1.0 \\
\hline SAQLI & $4.3(1.1)$ & $4.4(0.9)$ & $+0.8(1.0)$ & $+0.03(1.2)$ & -1.5 to +0.04 & 0.04 \\
\hline Neck size $(\mathrm{cm})$ & $46.2(2.6)$ & $47.0(2.6)$ & $+0.04(1.2)$ & $-0.06(1.4)$ & -0.7 to +0.9 & 0.8 \\
\hline Waist to hip ratio & $1.0(0.06)$ & $1.1(0.06)$ & $0(0.3)$ & $0(0.4)$ & -0.03 to +0.02 & 0.5 \\
\hline Impedance & $426.4(91.3)$ & $404.9(39.5)$ & $-3.8(28.4)$ & $+9.3(31.2)$ & -32.6 to +6.4 & 0.2 \\
\hline Fasting glucose (mmol/l) & $10.1(3.6)$ & $10.0(4.5)$ & $+0.3(2.1)$ & $-0.2(2.1)$ & -0.9 to +1.8 & 0.5 \\
\hline $\mathrm{HbAlc}(\%)$ & $8.5(1.8)$ & $8.4(1.9)$ & $-0.02(1.5)$ & $+0.1(0.7)$ & -0.6 to +0.9 & 0.7 \\
\hline Fasting plasma insulin (pmol/l) & $93.3(52.2)^{*}$ & $100.0(71.5)^{*}$ & $+1.3(1.6)$ & $+1.1(1.7)$ & -0.2 to +0.09 & 0.4 \\
\hline HOMA-\%S & $47.9(1.6)^{*}$ & $44.7(1.7)^{*}$ & $-1.5(2.3)^{*}$ & $-1.1(1.8)^{*}$ & -0.3 to +0.08 & 0.2 \\
\hline $\begin{array}{l}\text { M/l: euglycaemic clamp } \\
\left(1 / \mathrm{kg} / \mathrm{min}^{1000}\right)\end{array}$ & $26.5(14.4)$ & $27.8(17.9)$ & $+1.7(14.1)$ & $-5.7(14.8)$ & -1.8 to +0.3 & 0.2 \\
\hline Adiponectin $(\mu \mathrm{g} / \mathrm{ml})$ & $3.7(2.2)^{*}$ & $2.8(1.5)^{*}$ & $-1.1(1.2)^{*}$ & $-1.1(1.3)^{*}$ & -0.7 to +0.6 & 0.2 \\
\hline
\end{tabular}

CPAP, continuous positive airway pressure; $\mathrm{SaO}_{2}$, oxygen saturation; ESS, Epworth sleepiness score; MWT, Maintenance of Wakefulness test; $\mathrm{BMI}$, body mass index; SAQLI, Sleep Apnea Quality of Life Index; HbAlc, glycosylated haemoglobin; HOMA, homeostatic model assessment; M/I, quantity of glucose metabolised per unit of plasma insulin concentration (a measure of insulin sensitivity).

Data are shown as mean (SD).

${ }^{*}$ Geometric mean (SD).

There was no significant difference in any of the baseline data between the groups $(p=0.3-0.9)$ except for MWT $(p<0.01)$ and adiponectin ( $p=0.05)$. 
Table 2 Continuous positive airway pressure (CPAP) use

\begin{tabular}{lccl}
\hline & $\begin{array}{c}\text { Therapeutic CPAP } \\
(\mathbf{n}=19)\end{array}$ & $\begin{array}{c}\text { Placebo CPAP } \\
(\mathbf{n}=\mathbf{2 1})\end{array}$ & $\mathbf{p}$ Value \\
\hline Mean hours on nights used over last month & $3.6(2.8)$ & $3.3(3.0)$ & 0.8 \\
\% nights used over last month & $74.8(30.2)$ & $65.4(28.5)$ & 0.4 \\
Mean hours on nights used over last 3 months & $3.3(2.6)$ & $3.5(2.8)$ & 0.9 \\
\% nights used over last 3 months & $74.5(29.3)$ & $69.3(26.6)$ & 0.6 \\
$\%$ with compliance $<1$ h/night used & 26 & 14 & 0.4 \\
Mean hours on nights used in those with & $4.8(2.3)$ & $4.1(2.7)$ & 0.8 \\
$>1$ h/night compliance over last month & $4.4(2.0)$ & $4.2(2.6)$ & \\
Mean hours on nights used in those with & & & \\
$>1$ h/night compliance over last 3 months & & & \\
\hline
\end{tabular}

baseline and final euglycaemic clamps were not significantly different between groups. The geometric mean (SD) plasma insulin concentrations over the last $30 \mathrm{~min}$ of the baseline clamp were 1405 (239) $\mathrm{pmol} / \mathrm{l}$ in the therapeutic CPAP group and $1436(335) \mathrm{pmol} / \mathrm{l}$ in the placebo group $(\mathrm{p}=0.6)$, and in the repeat clamp geometric mean (SD) plasma insulin concentrations were 1453 (339) pmol/l and 1481 (327) pmol/ $\mathrm{l}$, respectively $(\mathrm{p}=0.8)$. Insulin sensitivity $(\mathrm{M} / \mathrm{I})$ is expressed as the quantity of glucose metabolised $(\mathrm{M})$ per unit of plasma insulin concentration (I), data obtained from the euglycaemic clamp. At the end of the study M/I had changed by +1.7 (14.1) in the therapeutic CPAP group compared with -5.7 (14.8) in the placebo CPAP group $(p=0.2)$. A positive change indicates an improvement in insulin resistance. HOMA-\%S changed by -1.5 (2.3) in the therapeutic group and -1.1 (1.8) in the placebo group $(p=0.2)$. Adiponectin did not change significantly following CPAP treatment in either of the groups (therapeutic - l.1 (1.2), placebo -1.1 (1.3), $\mathrm{p}=0.3$ ). The changes in HbAlc and adiponectin were found to be not normally distributed and therefore the two groups were compared again with the Mann-Whitney test, but this made no difference and the results remained non-significant $(p=0.3$ and 0.4 , respectively). There were no correlations between change in $\mathrm{M} / \mathrm{I}$ with change in weight, BMI, waist to hip ratio, bioelectrical impedance, HbAlc, HOMA-\%S, adiponectin or change in physical activity.

\section{Other blood tests}

There were no significant changes in any of the measures of fasting lipids or highly sensitive C-reactive protein over the 3month period in either of the two groups.

\section{Measures of body composition}

There was no significant change in any of the measures of BMI, waist to hip ratio, neck size or bioelectrical impedance over the 3-month period in either of the two groups (table 1).

\section{Actigraphy}

The average activity for the most active $10 \mathrm{~h}$ (M10, usually representing wakefulness) and the least active $5 \mathrm{~h}$ (L5, usually representing sleep) per day over a 7-day period were calculated. The activity levels were found to be highly variable and changes after CPAP in those receiving therapeutic CPAP did not reach statistical significance (M10, $\mathrm{p}=0.4 ; \mathrm{L} 5, \mathrm{p}=0.1$ ); mean (SD) change in M10: therapeutic CPAP +13.3 (68.3), placebo CPAP +0.7 (5.9), 95\% CI -43.0 to +17.7; mean (SD) change in L5: therapeutic CPAP -0.3 (0.8), placebo CPAP -0.4 (1.6), 95\% CI -1.0 to +0.8 arbitrary units/ 1000 .

\section{CPAP use}

There was no significant difference between the two groups in the mean number of hours for which CPAP was used on the nights it was used (table 2). In the therapeutic CPAP group there was no correlation between the hours of CPAP usage per night and the change in M/I, \%S-HOMA or HbAlc. Three subjects in the placebo CPAP group (14\%) and five in the therapeutic CPAP group (26\%) used CPAP for an average of $<\mathrm{l}$ h/night over the last $\mathrm{l}$ and 3 months. The main analyses were repeated with these poor compliers excluded (ie, per protocol) and the results remained non-significant with no suggestion of an improvement in the therapeutic group.

\section{DISCUSSION}

This double-blind randomised controlled trial of therapeutic and placebo CPAP for 3 months in men with type 2 diabetes and OSA has not shown any significant improvement in glycosylated haemoglobin or insulin resistance measured by euglycaemic clamp and HOMA. As anticipated, subjects receiving therapeutic CPAP experienced significant improvements in their subjective and objective sleepiness and sleep apnoea quality of life scores, similar to our previous studies, indicating that CPAP was effectively treating their OSA, but this was not accompanied by improvements in glycaemic control or insulin resistance.

There were no significant changes in any of the other variables measured, despite the clinical response of improvement in OSA. Adiponectin is an adipocyte derived peptide with insulin sensitising properties. It is decreased in adiposity and increases after weight reduction, and higher levels correlate with increased insulin sensitivity. ${ }^{20}$ A previous study found large and significant changes in adiponectin, together with significant changes in $\mathrm{M} / \mathrm{I}$, following treatment with pioglitazone. ${ }^{21}$ Adiponectin is therefore another marker, along with \% S and $\mathrm{M} / \mathrm{I}$, which can be used to determine if there is a change in insulin resistance but, in our study, it did not significantly change. The fact that neither the primary outcome measure of HbAlc nor any of the other variables associated with insulin resistance changed adds validity to the consistent findings of this study. The euglycaemic clamp is widely accepted as the gold standard for assessing insulin resistance; as markedly supraphysiological insulin concentrations are achieved, this method is applicable over a wide range of insulin sensitivities and glucose tolerances from normal to diabetes. ${ }^{22}$ Whatever the insulin resistance, agents that change the sensitivity tend to show effects with the euglycaemic clamp. The clamp data (Vmax effect) are concordant with the HOMA data (basal effect), ${ }^{82}$ so it is unlikely that a real change was observed with these two methods.

For comparison, a similar double-blind randomised controlled trial was carried out in 30 subjects with diet controlled type 2 diabetes to assess the effects of pioglitazone on glycaemic control and insulin resistance. ${ }^{21}$ Following 3 months of pioglitazone compared with placebo treatment, HbAlc significantly improved $(-0.6 \%, \mathrm{p}=0.003)$ as did HOMA-\%S $(+23 \%$, 
$\mathrm{p}=0.02), \mathrm{M} / \mathrm{I}\left(+12 \mathrm{l} / \mathrm{kg} / \mathrm{min}^{1000}, \mathrm{p}=0.009\right)$ and adiponectin $(3.8 \mathrm{ng} / \mathrm{ml}, \mathrm{p}=0.00004)$. These results show that larger significant improvements in all these measures are achieved with pioglitazone, whereas the small improvements in HbAlc and $\mathrm{M} / \mathrm{I}$ found in the CPAP group in our study are not statistically significant and are unlikely to be clinically significant. The validity characteristics of the euglycaemic clamp (mean (SD) of the measures of blood glucose concentrations over the last 20-30 $\mathrm{min}$ and SD of M/I) in our study were comparable to those in this pioglitazone study, as was the variation in the repeat measurements of HbAlc, HOMA-\%S and $\mathrm{M} / \mathrm{I}$ between the two time points.

The patients studied all had well established type 2 diabetes. The development of type 2 diabetes reflects a progressive decline in pancreatic $\beta$ cell function rather than increasing insulin resistance. ${ }^{23}$ The patients had a range of ages (2474 years) and were receiving different treatments for their diabetes; neither age nor diabetes treatment was significantly different between the two groups. In a comparison depending on a change following an intervention with within-subject comparisons being made, homogeneity of groups is not of the same importance as it is in a cross-sectional comparison. Wider recruitment can therefore be seen as an advantage.

Studies of drug treatment in patients with type 2 diabetes receiving different therapies have shown significant improvements in insulin resistance, typically within 3 months. ${ }^{24}{ }^{25}$ The use of CPAP for 3 months would therefore seem to be long enough for any changes in insulin resistance or glycaemic control to occur. It would be difficult to justify ethically giving placebo CPAP for longer than 3 months in this symptomatic group. It is possible that CPAP might be effective in a prediabetic group by improving insulin resistance via an improvement in the activity of the still functioning $\beta$ cells; this is an area for future research.

It could be argued that the mean CPAP compliance figures of $<4$ h use per night in our study might account for the lack of improvement in glycaemic and insulin resistance variables. If subjects had used their CPAP for longer, decreasing the number of apnoea-related arousals and the resultant sympathetic nervous system activation, would they have improved their insulin resistance? We do not think this is the case. First, the mean CPAP compliance was clearly great enough in the therapeutic group to improve the OSA, making a significant difference to sleepiness (measured by ESS, MWT and SAQLI) whereas the placebo group experienced no significant improvement. Since the sleepiness improved, the number of apnoearelated arousals was likely to have decreased together with the associated sympathetic nervous system hormone surges. Second, there was a range of mean compliance over the preceding month from zero use to $9.1 \mathrm{~h}$ /night, with poor and good compliers in both the therapeutic and placebo groups. A per protocol analysis of these good compliers did not change the results, neither could we could find a correlation between any of the measures of insulin resistance or HbAlc and CPAP compliance. We would have expected positive correlations if improvements in insulin resistance were associated with compliance. Indeed, even the study by Harsch et al ${ }^{13}$ showed no correlation between CPAP use and the improvements in insulin resistance. This is surprising, given that the treatment of OSA is thought to lead to improvements in insulin resistance via decreased sympathetic nervous system activation. We are clear that the outcome of our study is not due to lack of CPAP use.

The inclusion of a control group treated with placebo CPAP is particularly important in a study of insulin resistance. It is likely that glycaemic control and insulin resistance would be influenced by taking part in a study, regardless of the intervention, as people are more likely to modify their behaviour when they know they are being monitored. It would be impossible in an uncontrolled study to attribute any changes purely to the intervention concerned. There have been several studies published assessing the effect of CPAP on insulin resistance. None have used a control group, which leads to concern regarding the interpretation of the results. Harsch et al treated 40 patients with therapeutic CPAP and performed euglycaemic clamp studies prior to CPAP, after 2 days and after 3 months. ${ }^{13}$ A significant improvement in insulin sensitivity (the reciprocal of insulin resistance) was found after 2 days of CPAP treatment which was sustained at 3 months $(p=0.001)$. Mean BMI did not change during the study. The subgroup of patients with a BMI of $>30 \mathrm{~kg} / \mathrm{m}^{2}$ showed no significant change in insulin sensitivity at 2 days but a significant improvement at 3 months $(p=0.03)$. There was no correlation between improvement in insulin sensitivity and CPAP use. The investigators hypothesised that the early changes in insulin sensitivity after 2 days were due to improvements in sleep disordered breathing and associated decreases in nocturnal sympathetic drive, as well as improvements in the hypothalamic-pituitary-adrenal function due to improvements in sleep and hypoxia. The later changes in the patients with BMI $>30 \mathrm{~kg} / \mathrm{m}^{2}$ may have been due to changes in body fat distribution.

It has been noted previously that the clamp procedure itself increases sympathetic nervous system activity, presumably because patients are uncomfortable and anxious and this is likely to increase insulin resistance. ${ }^{26}$ Control patients who underwent a clamp procedure, but received only saline, had increases in plasma norepinephrine similar to those found in patients undergoing a euglycaemic clamp with insulin and glucose. By the time of the second clamp, patients are likely to have acclimatised to the situation and insulin resistance is hence reduced, ${ }^{27}$ and by the third clamp this acclimatisation would be greater. This effect makes the inclusion of a control group mandatory so that changes in insulin resistance are not falsely attributed to the intervention concerned. One study which assessed the change in insulin resistance in people with type 2 diabetes treated with either pioglitazone or placebo showed that insulin resistance (measured by euglycaemic clamp) improved in both groups after 3 months, although the improvement was greater in the pioglitazone group (41\% vs $10 \%$ in controls). ${ }^{21}$ The improvement in the control group was likely to be due, at least in part, to acclimatisation to the clamp procedure itself, as well as other factors such as better adherence to diet and medication or increased exercise. We did not see such improvements in either group in our study, possibly because the subjects were encouraged not to alter their diet or activity. It could be questioned whether the $18 \%$ and $31 \%$ improvement found in insulin resistance by Harsch et al ${ }^{13}$ after 2 days and 3 months respectively might not, in fact, be due to CPAP but to clamp acclimatisation, study effect or confounding variables.

The other studies published on this subject have had small numbers of patients $(n \leqslant 10)$ and have also not had control groups. A study of 10 subjects by Brooks et al ${ }^{12}$ showed a nonsignificant improvement in insulin sensitivity following 3 months of CPAP $(28 \%, p=0.06)$. A further study in nine subjects with type 2 diabetes by Harsch et al ${ }^{14}$ showed a significant improvement in insulin sensitivity after 3 months of CPAP $(42 \%, p=0.04) .{ }^{14}$ Both of these results could have been due to the effect of acclimatisation on the second clamp, study effect or biological variation in insulin sensitivity, as well as the confounders which affect insulin resistance. A study by Saarelainen et al $^{11}$ found no significant effect of treatment for 3 months with CPAP on euglycaemic clamp measures in 10 
subjects with OSA, ${ }^{11}$ as did a study by Smurra et al ${ }^{10}$ in 10 patients with OSA treated for 2 months with CPAP. Babu et a ${ }^{28}$ looked at HbAlc and $72 \mathrm{~h}$ continuous glucose monitoring before and after approximately 3 months of CPAP treatment. No significant improvement in overall HbAlc was found $(p=0.06)$, but HbAlc significantly improved in those patients in whom it was initially above $7 \%(\mathrm{p}=0.02$, likely to be due to regression to the mean) and there were some improvements in postprandial glucose levels $(p=0.05)$. Again, without a control group it is difficult to attribute these improvements in glycaemic control solely to CPAP and not to the effect of being monitored in a study.

In conclusion, OSA, insulin resistance and type 2 diabetes are all increasing in prevalence as population obesity levels increase. Our randomised placebo-controlled study adds evidence that CPAP does not improve insulin resistance and glycaemic control in men with established type 2 diabetes and OSA. Routine treatment of OSA in patients with type 2 diabetes is unlikely to result in improved diabetic control or a reduction in treatment requirements through a direct effect on insulin resistance.

\section{ACKNOWLEDGEMENTS}

The authors thank Joy Crosby who randomised all the patients and supervised their CPAP and the staff of the Oxford Centre for Diabetes, Endocrinology and Metabolism for their help with the euglycaemic clamps.

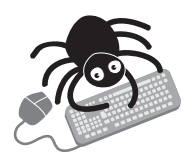

Additional details are provided in the online data supplement available at http://thorax.bmj.com/ supplemental.

Authors' affiliations

Sophie D West, Debby J Nicoll, John R Stradling, Sleep Unit, Oxford Centre for Respiratory Medicine, Churchill Hospital, Oxford, UK Tara M Wallace, David R Matthews, Oxford Centre for Diabetes, Endocrinology and Metabolism, Churchill Hospital, Oxford, UK

This study was funded by Diabetes UK and the Grand Charity.

Competing interests: None.

\section{REFERENCES}

1 Young T, Palta M, Dempsey J, et al. The occurrence of sleep-disordered breathing among middle-aged adults. N Engl J Med 1993;328:1230-5

2 Punjabi NM, Sorkin JD, Katzel LI, et al. L. Sleep-disordered breathing and insulin resistance in middle-aged and overweight men. Am J Respir Crit Care Med 2002; 165:677-82.

3 Ip MS, Lam B, Ng MM, et al. Obstructive sleep apnea is independently associated with insulin resistance. Am J Respir Crit Care Med 2002;165:670-6.

4 Reaven GM. Banting Lecture 1988. Role of insulin resistance in human disease. Diabetes 1988;37:1595-607.
5 Miyazaki Y, Glass L, Triplitt C, et al. A. Abdominal fat distribution and peripheral and hepatic insulin resistance in type 2 diabetes mellitus. Am J Physiol Endocrinol Metab 2002;283:E1135-43.

6 Punjabi NM, Ahmed MM, Polotsky VY, et al. Sleep-disordered breathing, glucose intolerance, and insulin resistance. Respir Physiol Neurobiol 2003;136:167-78.

7 Spiegel K, Leproult R, Van Cauter E. Impact of sleep debt on metabolic and endocrine function. Lancet 1999;354:1435-9.

8 Matthews DR, Hosker JP, Rudenski AS, et al. Homeostasis model assessment: insulin resistance and beta-cell function from fasting plasma glucose and insulin concentrations in man. Diabetologia 1985;28:412-9.

9 Defronzo RA, Tobin JD, Andres R. Glucose clamp technique: a method for quantifying insulin secretion and resistance. Am J Physiol 1979;237:E214-23.

10 Smurra M, Philip P, Taillard J, et al. CPAP treatment does not affect glucoseinsulin metabolism in sleep apneic patients. Sleep Med 2001;2:207-13.

11 Saarelainen S, Lahtela J, Kallonen E. Effect of nasal CPAP treatment on insulin sensitivity and plasma leptin. J Sleep Res 1997;6:146-7.

12 Brooks B, Cistulli PA, Borkman M, et al. Obstructive sleep apnea in obese noninsulin-dependent diabetic patients: effect of continuous positive airway pressure treatment on insulin responsiveness. J Clin Endocrinol Metab 1994;79:1681-5

13 Harsch IA, Schahin SP, Radespiel-Troger M, et al. Continuous positive airway pressure treatment rapidly improves insulin sensitivity in patients with obstructive sleep apnea syndrome. Am J Respir Crit Care Med 2004;169:156-62.

14 Harsch IA, Schahin SP, Bruckner K, et al. The effect of continuous positive airway pressure treatment on insulin sensitivity in patients with obstructive sleep apnoea syndrome and type 2 diabetes. Respiration 2004;71:252-9.

15 Jenkinson C, Davies RJ, Mullins R, et al. Comparison of therapeutic and subtherapeutic nasal continuous positive airway pressure for obstructive sleep apnoea: a randomised prospective parallel trial. Lancet 1999;353:2100-5.

16 Pepperell JC, Ramdassingh-Dow S, Crosthwaite N, et al. Ambulatory blood pressure after therapeutic and subtherapeutic nasal continuous positive airway pressure for obstructive sleep apnoea: a randomised parallel trial. Lancet 2002;359:204-10.

17 Bennett LS, Stradling JR, Davies RJO. A behavioural test to assess daytime sleepiness in obstructive sleep apnoea. J Sleep Res 1997;6:142-5.

18 Flemons WW, Reimer M. Measurement properties of the Calgary Sleep Apnoea Quality of Life index. Am J Respir Crit Care Med 2002;165:159-64.

19 Phillips P, Karrasch J, Scott R, et al. Acarbose improves glycemic control in overweight type 2 diabetic patients insufficiently treated with metformin. Diabetes Care 2003;26:269-73.

20 Tschritter O, Fritsche A, Thamer C, et al. Plasma adiponectin concentrations predict insulin sensitivity of both glucose and lipid metabolism. Diabetes 2003;52:239-43.

21 Wallace TM, Levy JC, Matthews DR. An increase in insulin sensitivity and basal beta-cell function in diabetic subjects treated with pioglitazone in a placebocontrolled randomized study. Diabet Med 2004;21:568-76.

22 Bonora E, Targher G, Alberiche $M$, et al. Homeostasis model assessment closely mirrors the glucose clamp technique in the assessment of insulin sensitivity: studies in subjects with various degrees of glucose tolerance and insulin sensitivity. Diabetes Care 2000;23:57-63.

23 UK Prospective Diabetes Study Group. UK Prospective Diabetes Study 16 Overview of 6 years' therapy of type II diabetes: a progressive disease. Diabetes 1995;44:1249-58.

24 Miyazaki Y, Mahankali A, Matsuda M, et al. Improved glycemic control and enhanced insulin sensitivity in type 2 diabetic subjects treated with pioglitazone. Diabetes Care 2001;24:710-9.

25 Yamasaki Y, Kawamori R, Wasada T, et al. Pioglitazone (AD-4833) ameliorates insulin resistance in patients with NIDDM. AD-4833 Glucose Clamp Study Group, Japan. Tohoku J Exp Med 1997;183:173-83.

26 Moan A, Hoieggen A, Nordby G, et al. The glucose clamp procedure activates the sympathetic nervous system even in the absence of hyperinsulinemia. J Clin Endocrinol Metab 1995;80:3151-4.

27 Punjabi NM. Improvement of metabolic function in sleep apnea: the power of positive pressure. Am J Respir Crit Care Med 2004; 169:139-40.

28 Babu AR, Herdegen J, Fogelfeld L, et al. Type 2 diabetes, glycemic control, and continuous positive airway pressure in obstructive sleep apnea. Arch Intern Med 28-2-, 2005; 165:447-52. 